\title{
Bipolar magnetic semiconducting behavior in VNbRuAI
}

Nag, Jadupati; Rani, Deepika; Kangsabanik, Jiban; Singh, Durgesh; Venkatesh, R.; Babu, P. D.; Suresh, K. G.; Alam, Aftab

Published in:

Physical Review B

Link to article, DOI:

10.1103/PhysRevB.104.134406

Publication date:

2021

Document Version

Publisher's PDF, also known as Version of record

Link back to DTU Orbit

Citation (APA):

Nag, J., Rani, D., Kangsabanik, J., Singh, D., Venkatesh, R., Babu, P. D., Suresh, K. G., \& Alam, A. (2021).

Bipolar magnetic semiconducting behavior in VNbRuAl. Physical Review B, 104(13), [134406].

https://doi.org/10.1103/PhysRevB.104.134406

\section{General rights}

Copyright and moral rights for the publications made accessible in the public portal are retained by the authors and/or other copyright owners and it is a condition of accessing publications that users recognise and abide by the legal requirements associated with these rights.

- Users may download and print one copy of any publication from the public portal for the purpose of private study or research.

- You may not further distribute the material or use it for any profit-making activity or commercial gain

- You may freely distribute the URL identifying the publication in the public portal

If you believe that this document breaches copyright please contact us providing details, and we will remove access to the work immediately and investigate your claim 


\title{
Bipolar magnetic semiconducting behavior in VNbRuAl
}

\author{
Jadupati Nag $\odot,{ }^{1}$ Deepika Rani, ${ }^{1, *}$ Jiban Kangsabanik $\odot,{ }^{1,2,{ }^{*}}$ Durgesh Singh $\odot,{ }^{1}$ R. Venkatesh $\odot,{ }^{3}$ P. D. Babu, \\ K. G. Suresh (10,,$^{1, \dagger}$ and Aftab Alam (10) ${ }^{1, \ddagger}$ \\ ${ }^{1}$ Department of Physics, Indian Institute of Technology Bombay, Mumbai 400076, India \\ ${ }^{2}$ Department of Physics, Technical University of Denmark (DTU), 2800 Kongens Lyngby, Denmark \\ ${ }^{3}$ UGC-DAE Consortium for Scientific Research, University Campus, Khandwa Road, Indore 452001, India \\ ${ }^{4} U G C-D A E$ Consortium for Scientific Research, Mumbai Centre, BARC Campus, Mumbai 400085, India
}

(Received 31 October 2020; revised 12 August 2021; accepted 30 September 2021; published 11 October 2021)

We report the theoretical prediction of a class of spintronic materials, namely, bipolar magnetic semiconductors (BMSs), also supported by our experimental data. BMSs possess a unique band structure with unequal band gaps for spin-up and spin-down channels and thus are useful for tunable spin-transport-based applications such as spin filters. The valence band and conduction band in BMSs approach the Fermi level through opposite spin channels and hence facilitate reversible spin polarization that is controllable via applied gate voltage. We report the quaternary Heusler alloy $\mathrm{VNbRuAl}$ to exactly possess the band structure of a BMS. A rigorous normal x-ray diffraction (XRD) fitting along with synchrotron XRD data confirms that this alloy crystallizes in the LiMgPdSn structure with partial B2 disorder. Transport measurements show a two-channel semiconducting behavior and a quasilinear dependence of negative magnetoresistance, indicating the possible semiconducting nature. The thermoelectric power data not only confirm the semiconducting nature but also give a strong indication of the BMS nature. Interestingly, VNbRuAl also appears to show features of a fully compensated ferrimagnetic (FCF) behavior with vanishing magnetization and significantly high ordering temperature $(>900 \mathrm{~K})$. Theoretical simulations of the special quasirandom structure predict partial B2 disorder to be mainly responsible for the coexistent BMS and FCF-like behavior. This study opens up the possibility of finding another class of materials for antiferromagnetic spintronics, with great significance for both fundamental and applied fronts.

DOI: 10.1103/PhysRevB.104.134406

\section{INTRODUCTION}

Heusler alloys are exhilarating due to their exotic physical properties with potential applications in areas such as spintronics, topological quantum matter, spin filters, etc. Many of them are known to have excellent stability, high Curie temperature $T_{\mathrm{C}}$, high spin polarization [1], and compatibility to grow thin films. Spintronic technology has several advantages over the conventional electronics [2-5]. One of the earliest classes of materials used was that of half-metallic ferromagnets (HMFs) [6,7]. Later, spin gapless semiconductors (SGSs) [8,9] were discovered. Magnetic semiconductors (MSs) [10] and spin semimetals [11] constitute other unique classes. A number of these classes of materials have been reported by our group in the past $[6-9,11]$. In this paper, we report yet another interesting class, namely, bipolar magnetic semiconductors (BMSs). BMSs bridge the gap between conventional semiconductors (CSs) and MSs. A CS has equal band gaps in both spin channels, whereas an MS possesses unequal values. BMSs acquire a band structure similar to that of MSs with a key difference that the valence band (VB) and conduction band $(\mathrm{CB})$ shift towards the Fermi level $\left(E_{F}\right)$ in opposite directions with respect to spin. This unique electronic feature

\footnotetext{
*These authors contributed equally to this work.

†suresh@phy.iitb.ac.in

‡aftab@phy.iitb.ac.in
}

enables BMSs to achieve reversible spin polarization which can be controlled via an applied gate voltage $V_{g}$. Schematics of the density of states (DOS) for CSs, MSs, and BMSs are shown in Fig. 1. BMSs can be characterized by three important energy parameters, $\Delta \epsilon_{1}, \Delta \epsilon_{2}$, and $\Delta \epsilon_{3} . \Delta \epsilon_{1}$ is the energy gap between the VB spin-up and CB spin-down channels, while $\Delta \epsilon_{2}\left(\Delta \epsilon_{3}\right)$ is the gap between the CB (VB) edges of the two spin channels, as depicted in Fig. 1(c). Also, $\Delta \epsilon_{1}+$ $\Delta \epsilon_{2}\left(=E_{g} \uparrow\right)$ and $\Delta \epsilon_{1}+\Delta \epsilon_{3}\left(=E_{g} \downarrow\right)$ are the band gaps for the spin-up and spin-down channels, respectively. One of the potential technologies where BMSs can be extremely useful is spin filters, where $E_{F}$ can be tuned into either the spin-up VB or the spin-down CB by varying $V_{g}$, and hence achieve a tunable spin polarization.

There exist a few theoretical proposals for BMSs in the literature [12-14]. The purpose of this paper is to first establish the BMS property in a quaternary Heusler alloy, VNbRuAl, using first-principles calculations. Next, we demonstrate some of the experimental evidence to confirm the necessary features of a BMS in VNbRuAl. In addition to being a BMS, experimental data show a unique nature of magnetism in this alloy which is consistent with a fully compensated ferrimagnet (FCF) with vanishing magnetization. Due to the B2 disorder, VNbRuAl does not follow the Slater-Pauling (SP) rule [15]. An FCF possesses several advantages over other spintronic materials, making it ideal for antiferromagnetic (AFM) spintronics. 


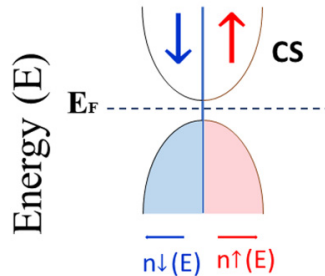

(a)

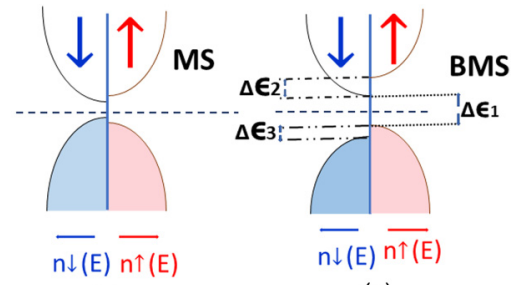

(c)
FIG. 1. Schematic density of states $n(E)$, for a typical (a) conventional semiconductor, (b) magnetic semiconductor, and (c) bipolar magnetic semiconductor

\section{EXPERIMENTAL AND COMPUTATIONAL DETAILS}

\section{A. Experimental details}

A polycrystalline sample of $\mathrm{VNbRuAl}$ was synthesized using arc melting in a high-purity argon environment of the constituents in stoichiometric proportion and with a purity of $99.99 \%$. To obtain a single phase, after melting, the ingot was annealed for 2 weeks at $800{ }^{\circ} \mathrm{C}$ in a sealed quartz tube, followed by furnace cooling. A room-temperature $\mathrm{x}$-ray diffraction (XRD) pattern was taken using $\mathrm{Cu}-\mathrm{K} \alpha$ radiation with the help of a Panalytical X-pert diffractometer. Crystal structure Rietveld refinement was done using FULLPROF Suite software [16]. Magnetization measurements at various temperatures were performed using a vibrating sample magnetometer (VSM) attached to the physical property measurement system (PPMS, Quantum Design) in fields up to $70 \mathrm{kOe}$. High-temperature magnetization measurements (300-900 K) were carried out using a superconducting quantum interference device magnetometer (magnetic property measurement system, Quantum Design). Temperature- and field-dependent resistivity measurements were carried out using the PPMS (DynaCool, Quantum Design) with the electrical transport option (ETO) in the traditional four-probe method, applying a $10 \mathrm{~mA}$ current at $18 \mathrm{~Hz}$ frequency. In order to understand the type of charge carriers and the variation of their density with temperature, Hall measurements were performed using the PPMS with the van der Pauw method again using $10 \mathrm{~mA}$ of current at $18 \mathrm{~Hz}$ frequency. Thermoelectric power (TEP) in zero applied magnetic field was measured using the differential dc sandwich method in a homemade setup in the temperature range 4-300 K [17].

\section{B. Computational details}

$A b$ initio calculations were performed using the spinresolved density functional theory (DFT) [18], as implemented within the Vienna ab initio simulation package (VASP) [19-21] with a projected augmented-wave (PAW) basis [22]. The pseudopotential formalism with the Perdew, Burke, and Ernzerhof (PBE) exchange-correlation functional [23] was used for primary electronic structure calculations. To perform the Brillouin zone integration within the tetrahedron method, a $24 \times 24 \times 24 \Gamma$-centered k-mesh was used. A plane-wave energy cutoff of $500 \mathrm{eV}$ was used for all the calculations. All the structures were fully relaxed with total energies (forces) converged to values less than $10^{-6} \mathrm{eV}(0.01 \mathrm{eV} / \AA)$. The
TABLE I. For VNbRuAl, relaxed lattice parameters $a_{0}$, total and atom-projected magnetic moments (in $\mu_{B}$ ), and relative energy $\Delta E$ of the three configurations with reference to the type-III configuration within the HSE06 functional.

\begin{tabular}{lrrrrrl}
\hline \hline Type & $a_{0}(\AA)$ & $m^{\mathrm{V}}$ & \multicolumn{1}{c}{$m^{\mathrm{Nb}}$} & \multicolumn{1}{c}{$m^{\mathrm{Ru}}$} & $m^{\text {total }}$ & $\Delta E(\mathrm{eV} /$ f.u. $)$ \\
\hline I & 6.26 & 2.53 & 0.38 & -0.28 & 2.6 & 1.24 \\
II & 6.19 & 0.00 & 0.00 & 0.00 & 0.00 & 0.7 \\
III & 6.24 & 2.72 & -0.10 & 0.18 & 2.80 & 0 \\
\hline \hline
\end{tabular}

PBE functional is known to underestimate the band gap of semiconducting materials. In the present case, because we are dealing with magnetic semiconductors, it is essential to go beyond the PBE calculation. To make a more accurate prediction, we employed the Heyd-Scuseria-Ernzerhof (HSE06) [24] functional and HSE06 $+G W$ approximation [25]. A 16-atom conventional cell and its symmetric $2 \times 2 \times 2$ supercell have been used while simulating ferromagnetic (FM) and different antiferromagnetic (AFM) configurations. To incorporate the B2 disorder, we have generated a 64-atom special quasirandom structure (SQS) [26]. An SQS is an ordered structure, known to mimic the random correlation accurately, for disordered compounds. Alloy Theoretic Automated Toolkit (ATAT) [27] was used to generate the SQSs. The generated SQSs perfectly mimic the random pair correlation functions up to third-nearest neighbors.

\section{THEORETICAL RESULTS}

VNbRuAl crystallizes in the LiMgPdSn prototype structure (space group $F \overline{4} 3 m$ ) with measured lattice parameter $6.15 \AA$. The structure can be seen as four interpenetrating fcc sublattices with Wyckoff positions $4 a, 4 b, 4 c$, and $4 d$. In general, for an $\mathrm{XX}^{\prime} \mathrm{YZ}$ alloy, there exist three energetically nondegenerate structural configurations (fixing the $\mathrm{Z}$ atom at the $4 a$ site). They are as follows: for type I, $\mathrm{X}$ at $4 c, \mathrm{X}^{\prime}$ at $4 d$, and $\mathrm{Y}$ at $4 b$; for type II, $\mathrm{X}$ at $4 b, \mathrm{X}^{\prime}$ at $4 d$, and $\mathrm{Y}$ at $4 c$; and for type III, $\mathrm{X}$ at $4 c, \mathrm{X}^{\prime}$ at $4 b$, and $\mathrm{Y}$ at $4 d$.

Using first-principles calculations, all three configurations were fully relaxed for VNbRuAl. Table I shows the relaxed lattice parameters, the total and atom-projected moments, and the relative energy differences among the type-I, typeII, and type-III configurations calculated using the hybrid HSE06 functional. The type-III configuration turns out to be the energetically most stable. The theoretically optimized lattice parameter for this configuration is $6.24 \AA$, which matches fairly well with the experimental value of $6.15 \AA$. The simulated net magnetization is $2.8 \mu_{B}$. Figure 2 shows the spin-polarized band structure and DOS for this configuration, which clearly confirms the BMS behavior. The DOS plot also highlights the three energy parameters $\Delta \epsilon_{1}, \Delta \epsilon_{2}$, and $\Delta \epsilon_{3}$, characterizing the BMS feature, as listed in Table II. For applications such as tunable spin polarization, it is desirable to achieve a small value of $\Delta \epsilon_{1}$ and large values of $E_{g} \uparrow$ and $E_{g} \downarrow$ (in comparison to $V_{g}$ ), which is indeed true for $\mathrm{VNbRuAl}$. We have also performed the $G W$ calculations [25] (going beyond the standard DFT framework), which again confirm the BMS 

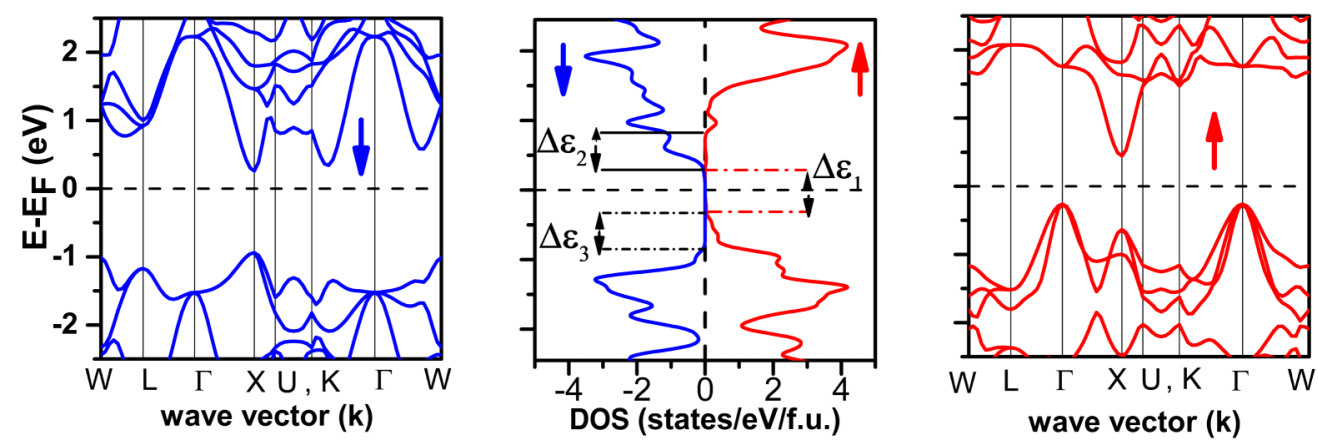

FIG. 2. For the pure type-III configuration of VNbRuAl, the spin-polarized band structure and density of states at the relaxed lattice parameter $a_{0}$ using the HSE06 functional.

nature of $\mathrm{VNbRuAl}$ with slightly different energy parameters. The detailed output of the HSE $+G W$ calculations is given in the Supplemental Material (SM) [28].

\section{EXPERIMENTAL RESULTS}

\section{A. Crystal structure}

Figure 3 shows the room-temperature XRD pattern of VNbRuAl along with the Rietveld refinement for configuration type III with 50\% disorder between the $\mathrm{V}$ and $\mathrm{Ru}$ atoms and a small amount of disorder between the $\mathrm{Nb}$ and $\mathrm{Al}$ atoms. Inset (ii) shows a zoomed-in view of the fitting near the (111) and (200) peaks. Inset (i) shows the refined data for pure configuration type III (with no disorder), which clearly do not fit well. For B2 disorder, we have considered several disorder variants, $5,10,25$, and $50 \%$, between the $\mathrm{V}$ and $\mathrm{Ru}$ sites and between the $\mathrm{Nb}$ and $\mathrm{Al}$ sites for all three configurations. However, the best fit was found with $50 \%$ antisite disorder between the $\mathrm{V}$ and $\mathrm{Ru}$ sites and $10 \%$ disorder between the $\mathrm{Nb}$ and $\mathrm{Al}$ sites in the three configurations. Although Fig. 3, inset (ii), and Figs. S4(b) and S4(d) of the SM [28] look similar, the best overall fitting was obtained only for configuration type III with the lowest $\chi^{2}$ value (2.64). The $\chi^{2}$ values for configuration types I, II, and III considering 50\% antisite disorder between the $\mathrm{V}$ and $\mathrm{Ru}$ sites and $10 \%$ disorder between the $\mathrm{Nb}$ and $\mathrm{Al}$ sites are 3.63, 3.09, and 2.64, respectively. Further details are shown in Sec. III A of the SM [28]. In order to probe the structure in more detail, we have carried out synchrotron XRD measurements as well. The synchrotron XRD data fully support the results of the structural analysis as obtained using the normal room-temperature XRD data. The detailed findings are presented in Secs. III A and III B of the SM [28].

TABLE II. For the pure type-III configuration of VNbRuAl, the relaxed lattice parameter (in $\AA$ ), energy parameters $\Delta \epsilon_{1}, \Delta \epsilon_{2}$, and $\Delta \epsilon_{3}$ (all in $\mathrm{eV}$ ), and band gaps for majority $\left(E_{g} \uparrow=\Delta \epsilon_{1}+\Delta \epsilon_{2}\right)$ and minority $\left(E_{g} \downarrow=\Delta \epsilon_{1}+\Delta \epsilon_{3}\right)$ spins using the HSE06 functional.

\begin{tabular}{lcccccc}
\hline \hline Type & $a_{0}$ & $\Delta \epsilon_{1}$ & $\Delta \epsilon_{2}$ & $\Delta \epsilon_{3}$ & $E_{g} \downarrow$ & $E_{g} \uparrow$ \\
\hline III & 6.24 & 0.65 & 0.52 & 0.47 & 1.12 & 1.17 \\
\hline \hline
\end{tabular}

\section{B. Magnetic properties}

Figures 4(a) and 4(b) show $M$-vs- $T$ and $M$-vs- $H$ curves for $\mathrm{VNbRuAl}$. Interestingly, the net magnetization turns out to be negligibly small $\left(\sim 10^{-3} \mu_{B}\right)$. The inset of Fig. 4(a) suggests an ordering temperature $>900 \mathrm{~K}$, much larger than that of other reported FCF materials [29]. The $M-H$ curve at $2 \mathrm{~K}$ shows a small, but nonzero hysteresis, with a small coercivity of 20 Oe [see inset (i) of Fig. 4(b)]. In particular, this curve does not saturate up to $90 \mathrm{kOe}$, indicating the compensated-ferrimagnetic-like behavior [30]. There can be various plausible reasons for such a vanishing moment, e.g., (i) compensated ferrimagnetism, (ii) long-range AFM, and (iii) $\mathrm{B} 2$ disorder.

\section{Transport properties 1. Resistivity}

Figure 4(c) shows the $T$ dependence of the longitudinal resistivity $\rho_{x x}$ at different fields [inset (i) of Fig. 4(c)]. $\rho_{x x}$

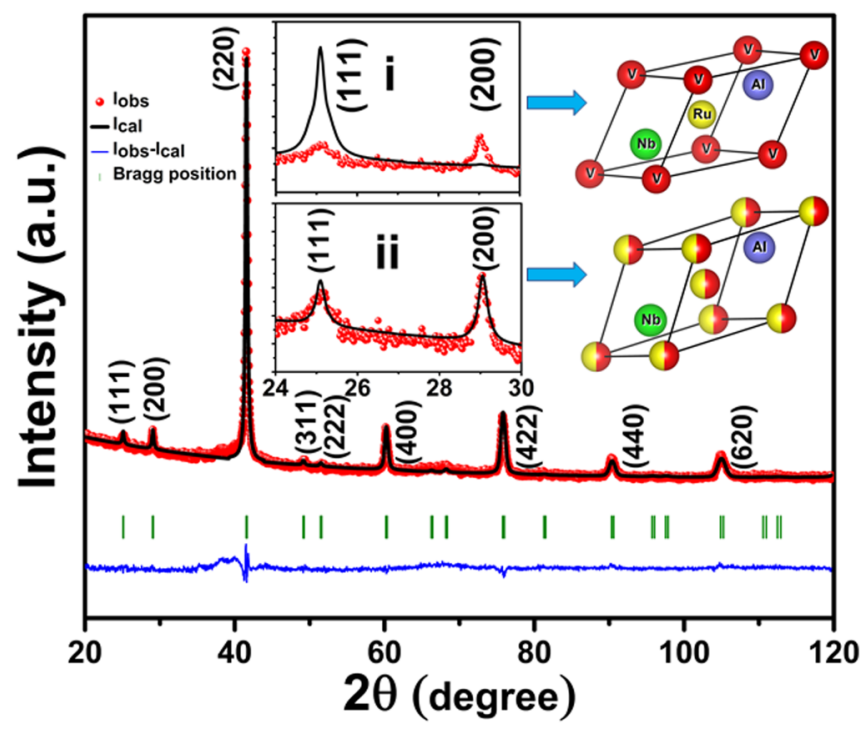

FIG. 3. Room-temperature powder XRD pattern of VNbRuAl, including the Rietveld-refined data for configuration type III with partial B2 disorder, i.e., 50\% disorder between the $\mathrm{V}$ and Ru sites and $<10 \%$ disorder between the $\mathrm{Nb}$ and $\mathrm{Al}$ sites. Insets (ii) and (i) show zoomed-in views near the (111) and (200) peaks with and without disorder, respectively. 

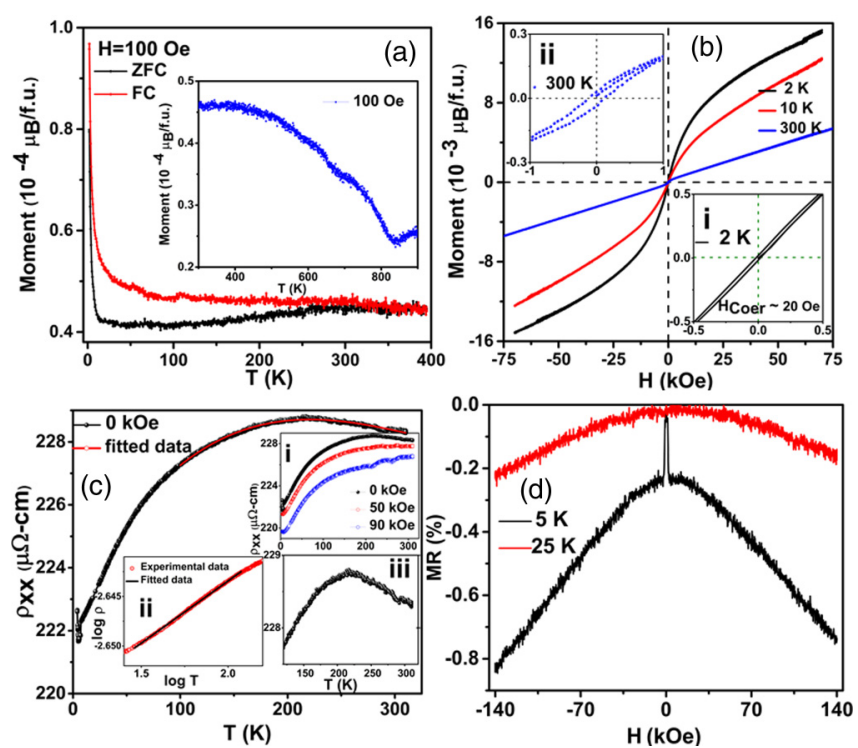

FIG. 4. For VNbRuAl, (a) $M$ vs $T$ in zero-field cooled (ZFC) and field cooled (FC) modes in $H=100 \mathrm{Oe}$. The inset shows the high- $T$ regime. (b) $M$ vs $H$ at 2, 10, and $300 \mathrm{~K}$. Insets (i) and (ii) display zoomed-in views at 2 and $300 \mathrm{~K}$, respectively. (c) Resistivity $\rho_{x x}$ vs $T$, along with a two-carrier model fit in zero field. Inset (i) shows $\rho_{x x}$ vs $T$ at three different fields, inset (ii) shows a $\log (\rho)$ vs $\log (T)$ plot fitted in the $T$ range $30-110 \mathrm{~K}$, and inset (iii) shows a zoomed-in view of $\rho$ in the high- $T$ regime $(>100 \mathrm{~K})$. (d) MR vs $H$ at 5 and $25 \mathrm{~K}$.

increases with $T$ in the low- $T$ region and goes through a maximum (at $\sim 220 \mathrm{~K}$ ) before falling as $T$ increases further towards $300 \mathrm{~K}$. The $T$ variation of $\rho_{x x}$ is quite similar to that seen in a few other Heusler systems $[31,32]$ with semiconducting behavior. The order of magnitude of $\rho$ in $\mathrm{VNbRuAl}$ is quite similar to that of other semiconducting materials [33]. At a glance, it appears that the low- $T$ region of $\rho_{x x}$ is metallic, and beyond $200 \mathrm{~K}$, it shows semiconductinglike behavior. The peak at $\sim 220 \mathrm{~K}$ [see inset (iii) of Fig. 4(c)] could be due to the competition between the positive $T$ coefficient of resistivity $(d \rho / d T>0)$ and the negative $d \rho / d T$ at higher $T$ due to semiconducting behavior. In many semiconducting materials with metalliclike behavior at low $T$ [31,32], the low- $T$ resistivity varies as $T^{\alpha}$ with $2<\alpha<3$. In order to check this, we have plotted $\log \left(\rho_{x x}\right)$ vs $\log (T)$ and fitted a straight line [see inset (ii) of Fig. 4(c)]. Such a fit is good only in the range of 30-110 K, and the value of $\alpha$ turns out to be 0.012 , which is surprisingly very small. An extremely slow increase in $\rho_{x x}$ with $T$ implies that the metallic character is quite weak. Thermally activated carriers (electrons and holes) of the semiconducting sample seem to be responsible for the behavior at higher $T$.

The conductivity data are fitted in the $T$ range of 120 $320 \mathrm{~K}$ with the modified two-carrier model [29,34],

$$
\sigma(T)=e\left(n_{e} \mu_{e}+n_{h} \mu_{h}\right)
$$

where $n_{i}=n_{i 0} e^{-\Delta E_{i} / k_{\mathrm{B}} T}(i=e, h)$ are the carrier concentrations of electrons and holes with mobilities $\mu_{i}$ and pseudogaps $\Delta E_{i} . \mu_{i}$ can be written as $\mu_{i}=\left(a_{i} T+b_{i}\right)^{-1}=\mu_{i 0} /\left(a_{i}^{\prime} T+\right.$ $1)$. Here, $a$ corresponds to carrier-phonon scattering, while $b$ arises from the mobility due to defects at $0 \mathrm{~K}$. Equation (1) then takes the form

$$
\sigma(T)=\left[A_{e}(T) e^{-\Delta E_{e} / k_{\mathrm{B}} T}+A_{h}(T) e^{-\Delta E_{h} / k_{\mathrm{B}} T}\right],
$$

where $A_{i}(T)=e n_{i 0} \mu_{i 0} /\left(a_{i}^{\prime} T+1\right)$. Equation (2) is used to fit the zero-field $\rho(T)$ data between 100 and $320 \mathrm{~K}$ [see Fig. 4(c)], and the energy gaps $\left(\Delta E_{i}\right)$ turn out to be 64.3 and $0.2 \mathrm{meV}$. The very fact that the two-carrier model describes the behavior of $\rho$ over such a wide $T$ range indicates that semiconducting behavior is dominant from $100 \mathrm{~K}$ itself. Interestingly, the zero-field $\rho$ peak at $\sim 220 \mathrm{~K}$ gets suppressed at higher field and shows a nearly saturated behavior with $T$ [35].

\section{Magnetoresistance}

Figure 4(d) shows the field dependence of magnetoresistance $(\mathrm{MR}), \operatorname{MR}(H)=[\rho(H)-\rho(0)] / \rho(0)$, at two different values of $T$. The magnitude of MR increases with $H$, and a sharp peaklike feature arises near $5 \mathrm{~K}$ within $\pm 2.5 \mathrm{kOe}$. The sensitivity of this peak to $H$ suggests its magnetic origin. There is a similar sharp rise in $\rho_{x x}$ below $6 \mathrm{~K}$ in zero-field $\rho$ (which gets suppressed at higher $H$ ) and a sharp rise in both ZFC and FC magnetization [see Fig. 4(a)]. These observations suggest some kind of spin reorientation below $6 \mathrm{~K}$; further experimental investigations such as neutron diffraction are required to get a better understanding of this. At or near $5 \mathrm{~K}$, the spins might be misaligned to give higher $\rho$, but when the field aligns them, $\rho$ drops sharply. With increasing $T$, the MR is reduced significantly and shows a quasilinear dependence on $H$, without any sign of saturation till $140 \mathrm{kOe}$, even at $5 \mathrm{~K}$ [see Fig. 4(d)]. The slope of this linear regime decreases with increasing $T$. Such a behavior reflects a gapped semiconductor [36], as reported in various systems by Abrikosov [37].

\section{Hall effect}

The Hall resistivity $\rho_{x y}$ vs $H$, at different $T$, shows a linear dependence (see Fig. S4 of the SM [28]). The carrier concentration $n$ at $5 \mathrm{~K}$ is $3.5 \times 10^{19} \mathrm{~cm}^{-3}$, which is comparable to that of other Heusler alloys having a semiconducting nature [1]. $n$ increases marginally (from $3.5 \times 10^{19}$ to $4.7 \times 10^{19}$ ) with increasing $T$, again indicating the robust semiconductinglike behavior of $\mathrm{VNbRuAl}$. The positive slope of the ordinary Hall coefficient suggests that holes are the majority charge carriers.

\section{Thermoelectric power}

The variation of the thermoelectric power $S$ with $T$ is shown in Fig. 5. The dependence of $S$ on $T$ is almost linear throughout the temperature range $(30-300 \mathrm{~K})$. The positive slope of the $S$-vs- $T$ plot corresponds to a purely hole-driven TEP. The linear behavior of $S$, which is very similar to that of other reported narrow-band-gap semiconductors [38,39], suggests the dominant contribution of diffusion thermopower to TEP. The magnitude of $S$ matches well with that of other reported semiconducting systems [40].

To understand the behavior of the density of states and the carrier density near the Fermi level, we have fitted the TEP data in the temperature range of 50-300 K with the equation $S_{d}=S_{0}+s T$, where $S_{d}$ denotes the diffusion thermopower, 


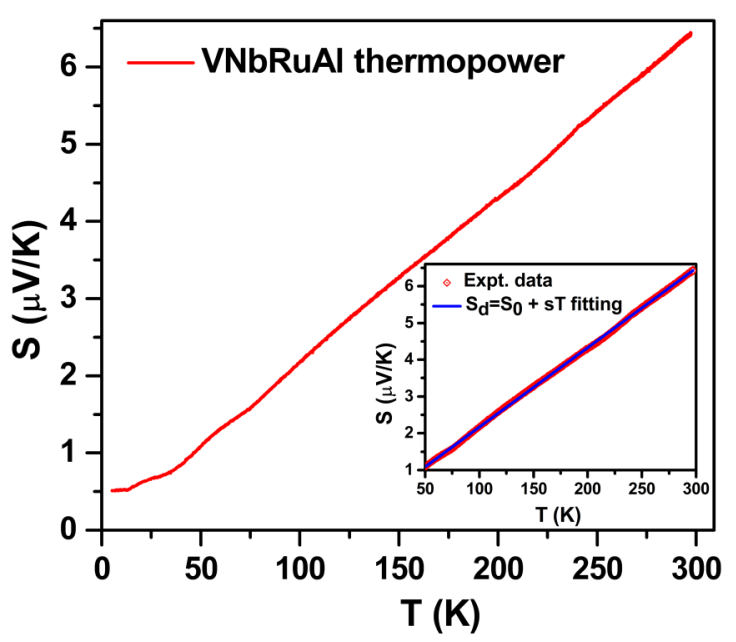

FIG. 5. Thermoelectric power $S$ vs $T$ for VNbRuAl. The inset shows a linear fitting to the $S$-vs- $T$ curve in the temperature range of 50-300 K. This illustrates the linear behavior of the diffusion thermopower $\left(S_{d}=S_{0}+s T\right)$ in this regime.

$S_{0}$ is a constant, and $s=\frac{\pi^{2} k_{B}^{2}}{3 e E_{F}}$. From this fitting we obtained $E_{F}$ to be $1.16 \mathrm{eV}$, and hole density $n=5.7 \times 10^{21} \mathrm{~cm}^{-3}$, which matches quite well with the semiconductor carrier density. For intrinsic semiconductors, $S$ has a large value with an increase in $T$ as $E_{F}$ lies within the band gap. However, for the nondegenerate semiconductors or BMSs (in which one of the spin channels has a very small gap, as seen in $\mathrm{VNbRuAl}$ ), the $E_{F}$ can move into the valence or conduction bands due to excitation (thermal or electric field induced) or impurity in one of the spin channels. This gives a small difference between the DOS above and below $E_{F}$ [41]. As a result of this, $S$ becomes small and increases linearly with $T$, as seen in VNbRuAl. Therefore the TEP data almost give direct evidence of the BMS nature of this alloy.

\section{EXPLAINING FCF-LIKE BEHAVIOR}

As mentioned earlier, magnetization measurements yield a negligibly small moment for $\mathrm{VNbRuAl}$, which may arise due to various reasons, e.g., (1) a unique AFM order and/or (2) complete or partial B2 disorder. To understand the exact reason, we first simulated a few distinct AFM configurations involving V atoms; see Fig. S3 of the SM [28]. As evident from Fig. S3 [28], the AFM-III phase lies only $10 \mathrm{meV}$ above the FM phase. This makes AFM-III almost equally probable to occur, giving rise to a zero moment. The band structure of AFM-III, however, does not show BMS behavior.

Taking a hint from the experimentally observed crystal structure, we further simulated the effect of B2 disorder (fully homogeneous disorder between $\mathrm{V}$ and $\mathrm{Ru}$ and between $\mathrm{Nb}$ and $\mathrm{Al}$ ) on the electronic and magnetic properties of $\mathrm{VN}$ bRuAl. For this, we generated special quasirandom structures (SQSs) [26] corresponding to three ordered configurations (see Table I). Table III shows the relative energies $\left(\Delta E_{\text {tot }}\right)$ among the three fully B2-disordered configurations, and the average spin- $\uparrow$ and spin- $\downarrow$ moments on $\mathrm{V}, \mathrm{Nb}$, and $\mathrm{Ru}$ atoms. Evidently, B2 disorder in type III is energetically most fa-
TABLE III. For VNbRuAl, relative energies $\Delta E_{\text {tot }}$ (in $\mathrm{eV} /$ f.u.) and average spin-up and spin-down moments (in $\mu_{B}$ ) at the $\mathrm{V}, \mathrm{Nb}$, and $\mathrm{Ru}$ atoms for homogeneous B2 disorder of three configurations, types I, II, and III. Type-III B2 disorder is the most stable.

\begin{tabular}{llcrcrrr}
\hline \hline Type & $\Delta \mathrm{E}_{\text {tot }}$ & $m_{\uparrow}^{\mathrm{V}}$ & $m_{\downarrow}^{\mathrm{V}}$ & $m_{\uparrow}^{\mathrm{Nb}}$ & $m_{\downarrow}^{\mathrm{Nb}}$ & \multicolumn{1}{c}{$m_{\uparrow}^{\mathrm{Ru}}$} & \multicolumn{1}{c}{$m_{\downarrow}^{\mathrm{Ru}}$} \\
\hline I & 0.434 & 0.00 & 0.00 & 0.00 & 0.00 & 0.00 & 0.00 \\
II & 0.136 & 0.00 & 0.00 & 0.00 & 0.00 & 0.00 & 0.00 \\
III & 0.0 & +0.73 & -0.62 & +0.01 & -0.02 & +0.04 & -0.01 \\
\hline \hline
\end{tabular}

vorable. Once again, in spite of giving a negligibly small net moment, the electronic structure of this configuration does not show BMS behavior.

We have then simulated a partial B2 disorder in the type III configuration where $\mathrm{V}$ and $\mathrm{Ru}$ atoms are fully mixed keeping $\mathrm{Nb}$ and $\mathrm{Al}$ sites fixed (it may be recalled that XRD fitting indicated a nominal, $10 \%$ disorder between $\mathrm{Nb}$ and $\mathrm{Al}$ ). The spin-polarized DOS is shown in Fig. 6 (the inset shows the SQS). Interestingly, this partially disordered phase gives the BMS and FCF (not simply AFM) behavior simultaneously. This is also energetically more stable (by $\sim 80 \mathrm{meV} /$ f.u.) than the completely disordered B2 phase. This results in the composite quantum property (BMS and FCF) of VNbRuAl.

\section{SUMMARY AND CONCLUSION}

In this paper, we report a class of spintronic materials which showcases the coexistence of two quantum phenomena, namely, a bipolar magnetic semiconductor and possibly fully compensated ferrimagnetism. Using a combined theoretical and experimental study, $\mathrm{VNbRuAl}$ is proposed to be a candidate system that exhibits this unique coexistence. It crystallizes in the cubic Heusler structure with partial B2 disorder, as revealed by our structural analysis using normal as well as synchrotron XRD. Resistivity data indicate two parallel channels of conduction, while MR and Hall data indicate a semiconducting nature. The thermoelectric power data not only support a semiconducting behavior but also give

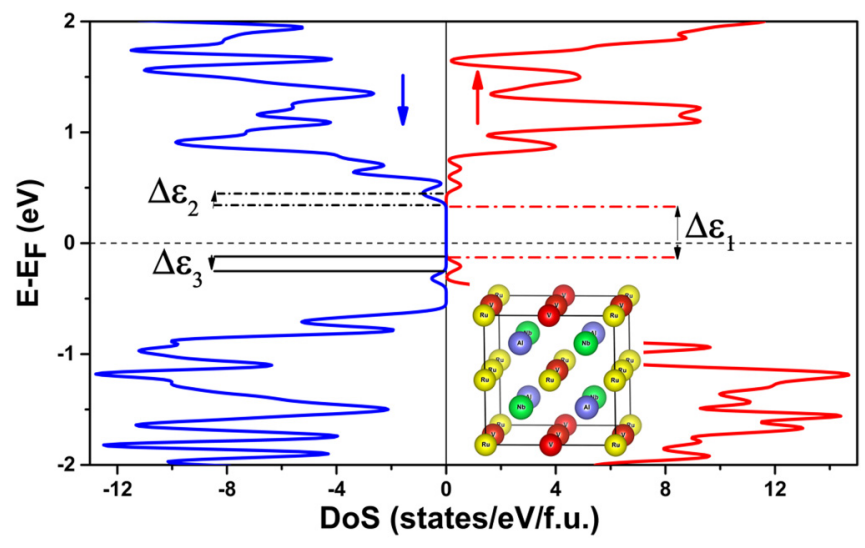

FIG. 6. Spin-polarized density of states for VNbRuAl with partial B2 disorder (i.e., complete disorder between $\mathrm{V}$ and $\mathrm{Ru}$, and no disorder involving $\mathrm{Nb}$ and $\mathrm{Al}$ sites). The inset shows the corresponding SQS. This structure supports both BMS behavior and the vanishing magnetization. 
a strong indication of BMS nature. Theoretical simulations predict partial B2 disorder (in $\mathrm{VNbRuAl}$ ) to be responsible for the coexistence of BMS and FCF properties. The vanishingly small moment and nonzero hysteresis can be attributed to the FCF-like behavior. The possibility of any such coexistence of different interesting properties (BMS, FCF, and high $T_{\mathrm{C}}$ ) in a single material opens up new opportunities for spintronics applications, e.g., room-temperature spin filters.

\section{ACKNOWLEDGMENTS}

J.N. and J.K. acknowledge the financial support provided by IIT Bombay. J.N. also thanks Dr. Velaga Srihari, ECXRD beamline, BL-11, Indus-2, RRCAT, for carrying out the anomalous X-ray diffraction measurements. A.A. acknowledges DST-SERB (Grant No. MTR/2019/000544) for funding this research.
[1] D. Rani, Enamullah, L. Bainsla, K. G. Suresh, and A. Alam, Phys. Rev. B 99, 104429 (2019).

[2] S. A. Wolf, D. D. Awschalom, R. A. Buhrman, J. M. Daughton, S. von Molnár, M. L. Roukes, A. Y. Chtchelkanova, and D. M. Treger, Science 294, 1488 (2001).

[3] I. Žutić, J. Fabian, and S. D. Sarma, Rev. Mod. Phys. 76, 323 (2004).

[4] C. Felser, G. H. Fecher, and B. Balke, Angew. Chem., Int. Ed. 46, 668 (2007).

[5] T. Graf, C. Felser, and S. S. Parkin, Prog. Solid State Chem. 39, 1 (2011).

[6] Y. Venkateswara, D. Rani, K. Suresh, and A. Alam, J. Magn. Magn. Mater. 502, 166536 (2020).

[7] Enamullah, Y. Venkateswara, S. Gupta, M. R. Varma, P. Singh, K. G. Suresh, and A. Alam, Phys. Rev. B 92, 224413 (2015).

[8] L. Bainsla, A. I. Mallick, M. M. Raja, A. K. Nigam, B. S. D. C. S. Varaprasad, Y. K. Takahashi, A. Alam, K. G. Suresh, and K. Hono, Phys. Rev. B 91, 104408 (2015).

[9] L. Bainsla, A. I. Mallick, M. M. Raja, A. A. Coelho, A. K. Nigam, D. D. Johnson, A. Alam, and K. G. Suresh, Phys. Rev. B 92, 045201 (2015).

[10] G. M. Stephen, G. Buda, M. E. Jamer, C. Lane, S. Kaprzyk, B. Barbiellini, D. Graf, L. H. Lewis, A. Bansil, and D. Heiman, J. Appl. Phys. (Melville, NY) 125, 123903 (2019).

[11] Y. Venkateswara, S. S. Samatham, P. D. Babu, K. G. Suresh, and A. Alam, Phys. Rev. B 100, 180404(R) (2019).

[12] X. Li, X. Wu, Z. Li, J. Yang, and J. Hou, Nanoscale 4, 5680 (2012).

[13] J. Zhang, X. Li, and J. Yang, J. Mater. Chem. C 3, 2563 (2015).

[14] J. Du, L. Feng, X. Wang, Z. Qin, Z. Cheng, and L. Wang, J. Alloys Compd. 710, 1 (2017).

[15] K. Özdoğan, E. Şaşığlu, and I. Galanakis, J. Appl. Phys. (Melville, NY) 113, 193903 (2013).

[16] J. Rodríguez-Carvajal, Physica B (Amsterdam) 192, 55 (1993).

[17] L. Sharath Chandra, A. Lakhani, D. Jain, S. Pandya, P. Vishwakarma, M. Gangrade, and V. Ganesan, Rev. Sci. Instrum. 79, 103907 (2008).

[18] P. Hohenberg and W. Kohn, Phys. Rev. 136, B864 (1964).

[19] G. Kresse and J. Furthmüller, Phys. Rev. B 54, 11169 (1996).

[20] G. Kresse and J. Furthmüller, Comput. Mater. Sci. 6, 15 (1996).

[21] G. Kresse and J. Hafner, Phys. Rev. B 47, 558 (1993).

[22] G. Kresse and D. Joubert, Phys. Rev. B 59, 1758 (1999).

[23] J. P. Perdew, K. Burke, and M. Ernzerhof, Phys. Rev. Lett. 77, 3865 (1996).
[24] A. V. Krukau, O. A. Vydrov, A. F. Izmaylov, and G. E. Scuseria, J. Chem. Phys. 125, 224106 (2006).

[25] L. Hedin, Phys. Rev. 139, A796 (1965).

[26] A. Zunger, S.-H. Wei, L. G. Ferreira, and J. E. Bernard, Phys. Rev. Lett. 65, 353 (1990).

[27] A. Van de Walle, P. Tiwary, M. De Jong, D. Olmsted, M. Asta, A. Dick, D. Shin, Y. Wang, L.-Q. Chen, and Z.-K. Liu, Calphad 42, 13 (2013).

[28] See Supplemental Material at http://link.aps.org/supplemental/ 10.1103/PhysRevB.104.134406 for details of a few theoretical and experimental results. In the theoretical results section, we present the electronic structure of the three different structural configurations (types I, II, and III) within the PBE functional and the spin-polarized density of states (DOS) using the HSE$G W$ approximation for the type-III configuration. We also present the energetics of various antiferromagnetic phases for the type-III configuration. We demonstrate the details of the XRD refinement, the synchrotron XRD data, and the data for Hall resistivity $\rho_{x y}$ vs field $H$ for $\mathrm{VNbRuAl}$ in the experimental results section.

[29] M. E. Jamer, Y. J. Wang, G. M. Stephen, I. J. McDonald, A. J. Grutter, G. E. Sterbinsky, D. A. Arena, J. A. Borchers, B. J. Kirby, L. H. Lewis, B. Barbiellini, A. Bansil, and D. Heiman, Phys. Rev. Appl. 7, 064036 (2017).

[30] G. M. Stephen, I. McDonald, B. Lejeune, L. H. Lewis, and D. Heiman, Appl. Phys. Lett. 109, 242401 (2016).

[31] M. E. Jamer, B. A. Assaf, T. Devakul, and D. Heiman, Appl. Phys. Lett. 103, 142403 (2013).

[32] Y. Nishino, M. Kato, S. Asano, K. Soda, M. Hayasaki, and U. Mizutani, Phys. Rev. Lett. 79, 1909 (1997).

[33] Y. Venkateswara, S. Gupta, S. S. Samatham, M. R. Varma, Enamullah, K. G. Suresh, and A. Alam, Phys. Rev. B 97, 054407 (2018).

[34] C. Kittel, Introduction to Solid State Physics, 7th ed. (Wiley, New York, 2007).

[35] Y. Shapira and T. Reed, Phys. Lett. A 35, 272 (1971).

[36] R. Xu, A. Husmann, T. Rosenbaum, M.-L. Saboungi, J. Enderby, and P. Littlewood, Nature (London) 390, 57 (1997).

[37] A. A. Abrikosov, Phys. Rev. B 58, 2788 (1998).

[38] M.-S. Lee and S. D. Mahanti, Phys. Rev. B 85, 165149 (2012).

[39] J. O. Sofo and G. D. Mahan, Phys. Rev. B 58, 15620 (1998).

[40] S. Ouardi, G. H. Fecher, C. Felser, and J. Kübler, Phys. Rev. Lett. 110, 100401 (2013).

[41] M. Markov, S. E. Rezaei, S. N. Sadeghi, K. Esfarjani, and M. Zebarjadi, Phys. Rev. Materials 3, 095401 (2019). 